\title{
Semi-analytical solution to MHD peristaltic flow of a Jeffrey fluid in presence of Joule heat effect by using multi-step differential transform method
}

\author{
Wahed M. Hasona ${ }^{1}$, Abdelhafeez El-Shekhipy ${ }^{2}$ and M. G. Ibrahim ${ }^{3}$ \\ ${ }^{1}$ Mathematics Department, Faculty of Science, Zagazig University, Zagazig, Egypt \\ ${ }^{2}$ Mathematics Department, Faculty of Science, Imam Abdulrahman Bin Faisal University, Al-Dammam, Kingdom of Saudi Arabia \\ ${ }^{3}$ Mathematics Department, Faculty of science, Al Azhar University, Cairo, Egypt
}

Received: 24 March 2018, Accepted: 30 July 2018

Published online: 30 April 2019.

\begin{abstract}
In this investigation, we portray the effect of inclined magnetic field on peristaltic flow of a Jeffrey fluid in presence of heat and mass transfer in an inclined symmetric or asymmetric channel. The Joule heating, Soret, Dufour and slip effects are taken into a consideration. The governing equations are converted from moving to fixed frame of reference and the resulting equations have then been simplified utilizing the assumptions of long wavelength and low but finite Reynolds number approximation. Semi analytical solutions have been obtained for the pressure gradient, temperature distribution, concentration distribution, longitudinal velocity and pressure rise by using the multi-steps differential transform method (Ms-DTM), a reliable and sturdy technique that improve accuracy and overcome drawbacks raised in using the standard differential transform method (DTM). This model is applicable to water transport from ground to upper branches of tall trees, petroleum industry, food industries and vegetable glycerin. In fact, the multi-step DTM is applicable to nonlinear models such as Non-Newtonian peristaltic fluid models which is more complicated and have a higher degree of non-linearity, in a direct way without using linearization, perturbation or restrictive assumptions.
\end{abstract}

Keywords: Peristaltic flow, Jeffrey Fluid, Joule Heating, Multi-step-differential transform method, concluding remarks.

\section{Introduction}

In the past few decades, substantial interest in studying the peristaltic flow in channels/tubes, because of it's a wide application and vital roles in geophysical, environmental, physiological and industrial processes. Slight such prominent processes contain spermatozoa transport in the ducts afferents of male reproductive tract, water transport from ground to upper branches of tall trees, blood pumps in heart lung machine, urine transport from kidney to bladder, chime movement in gastrointestinal tract, blood circulation in small blood vessels, sanitary and corrosive fluids transport, etc. (see [1-6]). Non-Newtonian Fluids has a singular characteristic; it show both properties of solid and liquid, as the relationship among the shear rate and the shear stress. As examples for application of non-Newtonian fluid: Food industries the petroleum industry oil refining industries, in processing industries such as paper production, hot rolling, wire drawing, glass-fiber production, etc.

Jeffrey fluid is a one subclass of non-Newtonian fluids which has been attracted much by the investigators, this fluid model is adequate for describe the characteristics of relaxation and retardation times. V.P. Rathod et al [7] addressed peristaltic flow of Jeffrey fluid with slip effects in an inclined channel. Effects of an endoscope and magnetic field on the peristalsis involving Jeffrey fluid is elucidates by Hayat et. al. [8]. Characteristics of Jeffrey fluid model for peristaltic flow of chime in small intestine with magnetic field is reported by Akbar et. al. [9]. Hayat et. al. [10] study the Impacts of 
constructive and destructive chemical reactions in magneto hydrodynamic (MHD) flow of Jeffrey liquid due to nonlinear radially stretched surface. Hussain et al [11] investigate the Heat transfer analysis in peristaltic flow of MHD Jeffrey fluid with variable thermal conductivity.

Joule heating occurs when the energy of an electric current is changed into heat as it flows through a resistance. There are many practical uses of Joule heating such as electric stoves and other electric heaters Soldering irons and cartridge heaters electric fuses electronic cigarettes usually work by Joule heating, vaporizing propylene glycol and vegetable glycerin, thermistors and resistance thermometers. Slip and Joule heating effects in mixed convection peristaltic transport of Nano fluid with Soret and Dufour effects is reported by Hayat et al [12]. Hayat et al [13] elucidates the radiative and Joule heating effects on peristaltic transport of dusty fluid in a channel with wall properties. Influence of Joule Heating on MHD Peristaltic Flow of a Nano fluid with Compliant Walls is reported by Reddy et al [14]. Influence of slip and Joule heating with radiation on MHD peristaltic blood flow with porous medium through a coaxial asymmetric vertical tapered channel blood flow analysis study is discussed by Abzal [15]. Hayat et al [16] addressed Joule heating and thermal radiation effects on peristalsis in curved configuration.

To be more specific, In transaction with heat and mass transfer problems, we address a phenomenon named by diffusion thermo effect (Duffour effect) in which an energy flux could be produced by the concentration gradients in addition to that generated by the temperature gradients, as well on otherwise mass fluxes could be produced by heat gradients which is renowned by thermal-diffusion effect (Sort effect) [21-22].

Because of the flow behavior of non-Newtonian fluids, the governing equations become more sophisticated to handle as supplemental nonlinear terms evidence in the equations of motion. Thus we turn to find the analytical solution for our model using a semi-analytical method named by Multi-step differential transform method (MsDTM). The DTM introduces promising approach form any applications in different domains of science. However, DTM has some disadvantages. By using the DTM, we obtain a series solution, actually a truncated series solution. This series solution does not offer the real behaviors of the problem but gives a pretty approximation to the true solution in a very small region. It is the purpose of this paper is to propose a reliable algorithm of the DTM. The new algorithm, multi-step DTM progressed in this paper, accelerates the convergence of the series solution over a large region and improves the accuracy of the DTM. For this, we apply the multi-step differential transform method, which provides the solution in terms of convergent series over a sequence of subintervals [17-20].

So far, no investigation has been made yet to elucidate the effects of Joule heating on peristaltic flow of a Jeffrey fluid in presence of heat and mass transfer under applying multi-step differential transform method, the velocity slip at the boundaries are taken into a consideration. Physical behaviors of different parameter are achieved and their salient features are established through figures for pressure rise, pressure gradient, velocity, temperature and concentration. To assure the numerical algorithm applied here, results are compared with good manners and found to be in excellent agreement. It is paramount to note that the results of this investigation are new and are published for the first time.

\section{Mathematical formulations}

Let us consider the peristaltic flow of non-Newtonian fluid (Jeffrey fluid) in a two-dimensional channel having widths $d_{1}$ and $d_{2}$, under the effect of a constant magnetic field. $\phi$ is the phase difference, $\phi$ varies in the range $0 \leq \phi \leq \phi$; $\phi=0$ corresponds to a symmetric channel with waves out of phase and for $\phi$ the waves are in phase. The geometry of the wall 
surface is defined as:

$$
\begin{aligned}
& Y=H_{1}=d_{1}+a_{1} \cos \left[\frac{2 \pi}{\lambda}(X-c t)\right] \quad \text { Upper wall } \\
& Y=H_{1}=-d_{2}-b_{1} \cos \left[\frac{2 \pi}{\lambda}(X-c t)+\phi\right] \text { Lower wall }
\end{aligned}
$$

Where $a_{1}$ and $b_{1}$ are the amplitudes of the waves, $d_{1}+d_{2}$ is the width of the channel, $c$ is the velocity of propagation, $t$ is the time and $X$ is the direction of wave propagation, Furthermore, $a_{1}, b_{1}, d_{1}, d_{2}$ and $\phi$ satisfy the condition.

$$
A_{1}^{2}+b_{1}^{2}+2 a_{1} b_{1} \cos \phi \leq\left(d_{1}+d_{2}\right)
$$

The governing equations of the flow under consideration can be expressed as follows $[1,6,15]$. The Continuity Equation:

$$
\frac{\partial U}{\partial X}+\frac{\partial V}{\partial Y}=0
$$

The Momentum Equations:

$$
\begin{aligned}
& \rho\left[\frac{\partial U}{\partial t}+U \frac{\partial U}{\partial X}+V \frac{\partial U}{\partial Y}\right]=-\frac{\partial P}{\partial X}+\frac{\partial S_{x x}}{\partial X}+\frac{\partial S_{x y}}{\partial Y}-\sigma B_{0}^{2} \cos \gamma(U \cos \gamma-V \sin \gamma)-\rho g \sin \alpha \\
& \rho\left[\frac{\partial V}{\partial t}+U \frac{\partial V}{\partial X}+V \frac{\partial V}{\partial Y}\right]=-\frac{\partial P}{\partial Y}+\frac{\partial S_{x y}}{\partial X}+\frac{\partial S_{y y}}{\partial Y}-\sigma B_{0}^{2} \sin \gamma(U \cos \gamma-V \sin \gamma)-\rho g \cos \alpha
\end{aligned}
$$

Heat equation yields:

$$
\begin{aligned}
C^{\prime}\left[\frac{\partial T}{\partial t}+U \frac{\partial T}{\partial X}+V \frac{\partial T}{\partial Y}\right]= & \frac{k^{\prime}}{\rho}+\left(\frac{\partial^{2} T}{\partial X^{2}}+\frac{\partial^{2} T}{\partial Y^{2}}\right)+\frac{\rho D_{m} k_{T}}{C_{s}}\left(\frac{\partial^{2} C}{\partial X^{2}}+\frac{\partial^{2} C}{\partial Y^{2}}\right)+v \frac{1}{1+\lambda_{1}}\left[1+\lambda_{2}\left(U \frac{\partial}{\partial X}+V \frac{\partial}{\partial Y}\right)\right] \\
& {\left[2\left(\frac{\partial U}{\partial X}\right)^{2}+2\left(\frac{\partial V}{\partial Y}\right)^{2}+\left(\frac{\partial U}{\partial X}+\frac{\partial V}{\partial Y}\right)^{2}\right]+\frac{\vec{J} \cdot \vec{J}}{\sigma} }
\end{aligned}
$$

Mass transfer equation yields:

$$
\left[\frac{\partial C}{\partial t}+U \frac{\partial C}{\partial X}+V \frac{\partial C}{\partial Y}\right]=D_{m}\left(\frac{\partial^{2} C}{\partial X^{2}}+\frac{\partial^{2} C}{\partial Y^{2}}\right)+\frac{D_{m} k_{T}}{T_{A}}\left(\frac{\partial^{2} T}{\partial X^{2}}+\frac{\partial^{2} T}{\partial Y^{2}}\right)
$$

Where $\langle U, V, 0\rangle$ are the velocity components in the laboratory frame $(X, Y), T$ is the temperature of the fluid, $C$ is the concentration of the fluid, $T$ is the mean value of $T 0$ and $T^{\circ}, C$ is the mean value of $C 0$ and $C^{\circ}$ Both the magnetic field and channel are inclined at angles $\gamma$ and $\alpha$ respectively, $g$ is the acceleration due to gravity, $\rho$ fluid density, $C^{\prime}$ concentration susceptibility, $K^{\prime}$ thermal diffusion ratio, $D_{m}$ mass diffusivity. The constitutive equation for the extra stress tensor $S$ is [1].

$$
S=\frac{\mu}{1+\lambda_{1}}\left(\dot{\gamma}+\lambda_{2} \ddot{\gamma}\right)
$$

Here $\lambda_{1}$ is the ratio of relaxation to retardation times, $\dot{\gamma}$ the shear rate, $\lambda_{2}$ the retardation time, and dots denote the differentiation with respect to time. The transformations between the laboratory and wave frames are given by $x=X-c t, y=Y, u=U-c, v=V$ and $p(x)=P(X, t)$. In which $(x, y),(u, v)$ and $p$ are the coordinates, velocity components and pressure in the wave frame. 
The dot product:

$$
\begin{aligned}
\vec{J} \cdot \vec{J} & =\sigma(\vec{v} \times \vec{B}) \cdot \sigma(\vec{v} \times \vec{B}) \\
& =\sigma^{2}(\vec{v} \times \vec{B}) \cdot(\vec{v} \times \vec{B}) \\
& =\sigma^{2}\left[\begin{array}{crr}
i & j & k \\
U & V & 0 \\
B_{0} \sin \gamma & B_{0} \cos \gamma & 0
\end{array}\right] \cdot\left[\begin{array}{ccr}
i & j & k \\
U & V & 0 \\
B_{0} \sin \gamma & B_{0} \cos \gamma & 0
\end{array}\right] \\
& =\sigma^{2} B^{2}(U \cos \gamma-V \sin \gamma)^{2}
\end{aligned}
$$

Defining following non-dimensional quantities:

$$
\begin{aligned}
& \bar{x}=\frac{x}{\lambda}, \bar{y}=\frac{y}{\mathrm{~d}_{1}}, \bar{u}=\frac{u}{\mathrm{c}}, \bar{v}=\frac{v}{\mathrm{c} \delta}, \bar{p}=\frac{d_{1}^{2}}{\mu \mathrm{c} \lambda}, \bar{t}=\frac{c t}{\lambda}, h_{1}=\frac{H_{1}}{d_{1}}, h_{2}=\frac{H_{2}}{d_{2}}, \\
& d=\frac{d_{2}}{d_{1}}, a=\frac{a_{1}}{d_{1}}, b=\frac{b_{1}}{d_{1}}, R_{e}=\frac{\rho c d_{1}}{\mu}, \theta=\frac{T-T_{0}}{T_{1}-T_{0}}, \varphi=\frac{C-C_{0}}{C_{1}-C_{0}}, P_{r}=\frac{c v C^{\prime}}{k^{\prime}} \\
& S_{c}=\frac{\mu}{\rho D_{m}}, \gamma=\frac{k^{\prime} d_{1}^{2}}{D_{m}}, S_{r}=\frac{\rho D_{m} k_{T}\left(T-T_{0}\right)}{\mu T_{A}\left(C_{1}-C_{0}\right)}, D_{f}=\frac{\rho D_{m} k_{T}\left(C-C_{0}\right)}{\mu C_{s} C^{\prime}\left(T_{1}-T_{0}\right)} \\
& r e_{c}=\frac{c^{2}}{C^{\prime}\left(T_{1}-T_{0}\right)}, M^{2}=\frac{\sigma_{0} B_{0} d_{1}^{2}}{\mu}, F_{r}=\frac{c^{2}}{g d_{1}}, \bar{S}=\frac{S d_{1}}{\mu c}, C_{1}=\frac{k_{1} d_{1}^{2} C}{D_{m}\left(C-C_{0}\right)} .
\end{aligned}
$$

Where $R_{e}$ is the Reynolds number, $\delta$ is the dimensionless wave number, $P_{r}$ is the Prandtl number $S_{c}$ is the Schmidt number, $S_{r}$ is the Soret number, $D_{f}$ Dufour number, $M$ Hartman number, $F_{r}$ Froude number and $E_{c}$ is the Eckert number. Writing the stream function $\psi=\psi(x, y)$, which is related to $\mathrm{u}$ and $v$ by the relations $u=\frac{\partial \psi}{\partial y}, v=-\frac{\partial \psi}{\partial x}$, and by substituting with equations (9), (11) and (??) into Eqs. (4-7) (After dropping bars) yield.

$$
\begin{gathered}
\delta R_{e}\left[\psi_{y} \psi_{x y}-\psi_{x} \psi_{y y}\right]=-\frac{\partial p}{\partial x}+\delta^{2} \frac{\partial S_{x x}}{\partial x}+\frac{\partial S_{x y}}{\partial y}-M^{2} \cos \gamma\left(\psi_{y}+1\right) \cos \gamma+\delta \psi_{x} \sin \gamma+\frac{R_{e}}{F_{r}} \sin \alpha \\
\delta R_{e}\left[-\psi_{y} \psi_{x x}+\psi_{y} \psi_{x y}\right]=-\frac{\partial p}{\partial y}+\delta^{2} \frac{\partial S_{y x}}{\partial x}+\delta \frac{\partial S_{x y}}{\partial y}-\delta M^{2} \sin \gamma\left(\psi_{y}+1\right) \cos \gamma+\delta \psi_{x} \sin \gamma-\frac{R_{e}}{F_{r}} \cos \alpha \\
\delta R_{e}\left[\psi_{y} \theta_{x}-\psi_{x} \theta_{y}\right]=-\frac{1}{P_{r}}\left[\theta_{y y}+\delta^{2} \theta_{x x}\right]+\frac{1}{1+\lambda_{1}}\left(\left(1+\frac{\lambda_{2} c \delta}{d_{1}}\left(\psi_{y} \frac{\partial}{\partial x}-\psi_{x} \frac{\partial}{\partial y}\right)\right)\left(4 \delta^{2} \psi_{x y}^{2}+\left(\psi_{y y}-\delta^{2} \psi_{x x}\right)\right)\right) \\
-M^{2} E_{c} \cos ^{2} \gamma\left(\psi_{y}+1\right)^{2} \\
\delta R_{e}\left[\psi_{y} \varphi_{x}-\psi_{x} \varphi_{y}\right]=\frac{1}{S_{c}}\left[\delta^{2} \varphi_{x x}+\varphi_{y y}\right]+S_{r}\left[\delta^{2} \theta_{x x}+\theta_{y y}\right] .
\end{gathered}
$$

Where equation (4) is satisfied identically and,

$$
\begin{gathered}
S_{x x}=\frac{2 \delta}{1+\lambda_{1}}\left[1+\frac{c \delta \lambda_{2}}{d_{1}}\left(\psi_{y} \frac{\partial}{\partial x}-\psi_{x} \frac{\partial}{\partial y}\right)\right] \psi_{x y} \\
S_{x y}=\frac{2 \delta}{1+\lambda_{1}}\left[1+\frac{c \delta \lambda_{2}}{d_{1}}\left(\psi_{y} \frac{\partial}{\partial x}-\psi_{x} \frac{\partial}{\partial y}\right)\right]\left[\psi_{y y-} \delta^{2} \psi_{x x}\right]
\end{gathered}
$$




$$
S_{y y}=\frac{2 \delta}{1+\lambda_{1}}\left[1+\frac{c \delta \lambda_{2}}{d_{1}}\left(\psi_{y} \frac{\partial}{\partial x}-\psi_{x} \frac{\partial}{\partial y}\right)\right] \psi_{x y} .
$$

Then by adopting the long wavelength $(\delta \ll 1)$, low Reynolds number process, equation (12-15) becomes

$$
\begin{gathered}
\frac{\partial p}{\partial x}+\frac{\partial}{\partial y}\left(\frac{1}{1+\lambda_{1}} \frac{\partial^{2} \psi}{\partial y^{2}}\right)-M^{2} \cos ^{2} \gamma\left(\psi_{y}+1\right)+\frac{R_{e}}{F_{r}} \sin \alpha=0, \\
\frac{\partial p}{\partial y}=0, \\
\frac{1}{P_{r}} \frac{\partial^{2} \theta}{\partial y^{2}}+\frac{E_{c}}{1+\lambda_{1}}\left(\frac{\partial^{2} \psi}{\partial y^{2}}\right)-D_{f} \frac{\partial^{2} \varphi}{\partial y^{2}}-M E_{c} \cos ^{2} \gamma\left(\frac{\partial \psi}{\partial y}+1\right)^{2}=0, \\
\frac{1}{S_{c}}+\frac{\partial^{2} \varphi}{\partial y^{2}}+S_{r} \frac{\partial^{2} \theta}{\partial y^{2}} .
\end{gathered}
$$

Implies that $p$ is not a function in $y$ and eliminating pressure above Eqns.

$$
\frac{\partial^{2}}{\partial y^{2}}\left(\frac{1}{1+\lambda_{1}} \frac{\partial^{2} \psi}{\partial y^{2}}\right)-M^{2} \cos ^{2} \gamma \frac{\partial^{2} \psi}{\partial y^{2}}=0,
$$

The convenient boundary conditions can be put into the following forms:

$$
\begin{gathered}
\psi=\frac{q}{2}, \varphi=0, \theta=0, \frac{\partial \psi}{\partial y}+L \frac{\partial^{2} \psi}{\partial y^{2}}=-1, \text { at } y=h_{1}=1+\operatorname{acos} 2 \pi x, \\
\psi=-\frac{q}{2}, \varphi=1, \theta=1, \frac{\partial \psi}{\partial y}-L \frac{\partial^{2} \psi}{\partial y^{2}}=-1, \text { at } y=h_{2}=-d-b \cos (2 \pi x+\phi) .
\end{gathered}
$$

Where $L$ the non-dimensional is slipping parameter and $q$ is the flux in the wave frame. The dimensional time mean flow rate $Q$ in the laboratory frame is related to $q$ through the relation $[1,13]$

$$
Q=q+1+d
$$

\section{Differential transformation method}

Consider a general equation of $\mathrm{n}^{\text {th }}$ order ordinary differential equation [19]

$$
y\left(t, f, f^{\prime}, \ldots, f^{(n)}\right)=0 .
$$

Subject to the initial equations

$$
f^{(k)}(0)=d_{k}, k=0, \ldots, n-1 .
$$

To demonstrate the differential transformation method (DTM) for solving differential equations, the basic definitions of differential transformation are introduced as follows. Let $f(t)$ be analytic in a domain $D$ and let $t=t^{\circ}$ represent any point in $D$. The function $f(t)$ is then represented by one power series whose centre is located at $\mathrm{t}^{\circ}$. The differential 
transformation of the $k-t h$ derivative of a function $f(t)$ is defined as the following:

$$
F(k)=\left(\frac{1}{k !}\right)\left[\left(\frac{d^{(k)} f(t)}{d t^{(k)}}\right)\right]_{\left(t=t^{0}\right)}, \forall t \in D .
$$

And the inverse transformation of $F(k)$ can take the form

$$
f(t)=\sum_{k=0}^{\infty} F(k)\left(t-t_{0}\right)^{(k)}, \forall t \in D .
$$

In fact, from Eq. (27) and (28), we obtain

$$
f(t)=\sum_{k=0}^{\infty} \frac{\left(t-t_{0}\right)^{(k)}}{k !}\left(\frac{d^{(k)} y(t)}{d t^{(k)}}\right)_{t=t_{0}}, \forall t \in D
$$

Eq. (28) implies that the concept of differential transformation is derived from the Taylor series expansion. Form the definitions of (27) and (28); it is easy to prove that the functions comply with the following basic mathematics operations (see Table 1). In real applications, the function $f(t)$ is expressed by a finite series and (29) can be written as:

$$
f(t)=\sum_{k=0}^{N} F(k)\left(t-t_{0}\right)^{(k)}, \forall t \in D .
$$

Eq. (30) implies that $\sum_{k=N+1}^{\infty} F(k)\left(t-t_{0}\right)^{(k)}$ is negligibly small. The following table show that the transformation for some functions and relation by using differential transformation method.

Table 1: Operations of the one dimensional differential transform.

\begin{tabular}{|ll|}
\hline Original function & Transformed function \\
\hline$f(t)=g(t)+h(t)$ & $F(k)=G(k)+H(k)$ \\
\hline$f(t)=\alpha g(t)$ & $F(k)=\alpha G(k)$ \\
\hline$f(t)=g(t) h(t)$ & $F(k)=\sum_{l=0}^{k} G(l) H(k-l)$ \\
\hline$f(t)=\frac{d g(t)}{d t}$ & $F(k)=(k+l) G(k+l)$ \\
\hline$f(t)=\frac{d^{n} g(t)}{d t^{n}}$ & $F(k)=\frac{(k+l) !}{k !} G(k+n)$ \\
\hline$f(t)=u(t) v(t) w(t)$ & $F(k)=\sum_{l=0}^{k} \sum_{r=0}^{k-l} U(l) V(l) w(k-r-l)$ \\
\hline
\end{tabular}

\section{Multi-steps differential transformation method}

The multi-step DTM is treated as an algorithm in a sequence of intervals for finding accurate approximate solutions for systems of differential equations.

Suppose $[0, T]$ is the interval over which we want to find the solution for a system of equations(27 - 29). In actual applications of the DTM, the approximate solution for a system of equations can be expressed by the finite series

$$
f(t)=\sum_{k=0}^{N} a_{(k)} t^{(k)}, t \in[0, T]
$$

The multi-steps approach introduces a new idea for constructing the approximate solution. Assume that the interval $[0, T]$ is divided into $M$ sub intervals $\left[t_{m-1}, t_{m}\right], m=1,2, \ldots, M$ of equal step size $h=\frac{T}{M}$ by using the nodes $t_{m}=m h$. The main 
ideas of the Multi-step DTM are as follows. First, we apply the DTM to a system of equations $(27-29)$ over the interval $[0, T]$ we will obtain the following approximate solution,

$$
f_{1}(t)=\sum_{k=0}^{N} a_{1 n} t^{k}, t \in\left[0, t_{1}\right]
$$

Using the initial conditions $f^{(k)}(0)=C_{k}$ Form $m \geq 2$ and at each sub interval $\left[t_{m-1}, t_{m}\right]$ we will use the initial conditions $f_{m}^{(k)}\left(t_{m=1}\right)=f_{m=1}^{(k)}\left(t_{m=1}\right)$ and apply the DTM to Eqs. $(25-27)$ over the interval $\left[t_{m=1}, t_{m}\right]$, where $t^{\circ}$ in Eq. (26) is replaced by $t_{m-1}$ the process is repeated and generates a sequence of approximate solution sum. $f_{m}(t), m=1,2, \ldots, M$ for the solution $f(t)$.

$$
f_{m}(t)=\sum_{k=0}^{N} a_{m k}\left(t-t_{\{m-1\}}\right)^{2}, t \in\left[t_{m}, t_{m-1}\right]
$$

\section{Analytical solutions by means of the Multi-step DTM}

Now, we apply the differential transform method properties as table 1 to Eqns. $(21-23)$ For finding $\psi(x, y)$, $\theta(x, y), \varphi(x, y)$ and $p(x, y)$, then we have

$$
\begin{gathered}
\left(\frac{[k+1][k+2][k+3][k+4]}{1+\lambda_{1}}\right) \Psi[k+4]-M^{2} \cos ^{2} \gamma[k+1][k+2] \Psi[k+2]=0, \\
{[k+1][k+2]\left[\left(\frac{1}{P_{r}}\right) \Theta[k+2]+D_{f} \Phi[k+2]\right]+\left(\frac{E_{c}}{1+\lambda_{1}}\right) \sum_{r=0}^{k}[r+1][k-r+1][k-r+2] \Psi[r+1] \Psi[k-r+2]} \\
-M^{2} \cos ^{2} \gamma E_{c}\left(\delta(k, 0)+2[k+1] \Psi[k+1]+\sum_{r=0}^{k}[r+1][k-r+1] \Psi[r+1] \Psi[k-r+1]\right)=0, \\
\frac{1}{S_{c}}[k+1][k+2] \Phi[k+2]=-S_{r}[k+1][k+2] \Theta[k+2] .
\end{gathered}
$$

With the associated boundary conditions.

$$
\begin{aligned}
& \sum_{k=0}^{n} \Psi_{k}\left[h_{2}(x)-h_{1}(x)\right]^{k}=\frac{q}{2}, \sum_{k=0}^{n} \Theta_{k}\left[h_{2}(x)-h_{1}(x)\right]^{k}=0, \sum_{k=0}^{n} \Phi_{k}\left[h_{2}(x)-h_{1}(x)\right]^{k}=0, \\
& \sum_{k=1}^{n} k \Psi_{k}\left[h_{2}(x)-h_{1}(x)\right]^{k-1}+L \sum_{k=2}^{n} k(k-1) \Psi_{k}\left[h_{2}(x)-h_{1}(x)\right]^{k-2}=-1 \quad \text { at } y=h 0 . \\
& \sum_{k=0}^{n} \Psi_{k}\left[h_{2}(x)-h_{1}(x)\right]^{k}=\frac{-q}{2}, \sum_{k=0}^{n} \Theta_{k}\left[h_{2}(x)-h_{1}(x)\right]^{k}=1, \sum_{k=0}^{n} \Phi_{k}\left[h_{2}(x)-h_{1}(x)\right]^{k}=1, \\
& \sum_{k=1}^{n} k \Psi_{k}\left[h_{2}(x)-h_{1}(x)\right]^{k-1}-L \sum_{k=2}^{n} k(k-1) \Psi_{k}\left[h_{2}(x)-h_{1}(x)\right]^{k-2}=-1 \quad \text { at } y=h_{2} .
\end{aligned}
$$

\section{Graphical results and discussions}

Here the non-linear analysis is computed for the Ms-DTM. The characteristics of several emerging parameters on the distributions of pressure rise and pressure gradient as well as velocity, temperature and concentration fields are plotted in the Figs. 1-25. 


\subsection{Pressure rise}

Figs. 1-4 are ploted to see the effects of various values of the angle of inclination $\gamma$, Jeffrey parameter $\lambda 0$, Froude number $F_{r}$ and Hartmann number $M$ on pressure rise. It is observed that there is a linear relationship between $\Delta \mathrm{P}$ and $Q$. Values for which $\Delta \mathrm{P}>0$ and $Q<0$ is known as the retrograde pumping or backward pumping region. For $\Delta \mathrm{P}>0 ; 0<Q<Q^{\circ}$ indicates peristaltic pumping region, at $Q=Q^{\circ}>0 ; \Delta \mathrm{P}=0$ this refer to the case of free pumping. Finally, the region was $\Delta \mathrm{P}<0$ and $Q>Q^{\circ}>0$ is called the co-pumping or the augmented region.

- Influences of $\lambda 0$ and $\gamma$ are portrayed in Figs 1-2. Here it is observed that the pumping rate decrease by increasing $\lambda 0$ and $\gamma$ in the retrograde and peristaltic regions till a certain value $Q=Q^{*}$, after which $\Delta \mathrm{P}$ increases with $Q>Q^{*}$. It is noticed that the peristaltic pumping region becomes lightly wider with the decrease of $\lambda 0$ and $\gamma$.

- Fig 3. Describe the behavior of $F_{r}$ on pressure rise $\Delta P$, it's clear that pressure rise decreases in all pumping region with an increase in Froude number $F_{r}$.

- The opposite effect of $\lambda 0$ can be observed with increasing in Hartmann number on the pumping rate in Fig 4.

\subsection{Pressure gradient}

Figs. 5-8 are affirmed to visualize the influence of pressure gradient $\frac{d P}{d x}$ for various values of Jeffrey parameter $\lambda 0$, Froude number $F_{r}$, Hartmann number $\mathrm{M}$ and the angle of inclination $\gamma$.

- Fig. 5 Show that for high Jeffrey parameter $\lambda 0$ the resistance to the flow in the central part of the channel decreases?

- Fig. 6 implies that for the Froude number $F_{r}$ the resistances to the flow all over the channel decreases.

- The pressure gradient for different values of $\mathrm{M}$ and against $\mathrm{x}$ is plotted in Figs. 7-8. It is shown that for $x \in[0,0.23]$ and $x \in[0.6,1]$, the pressure gradient is small, i.e. the flow can easily pass without imposition of a large pressure gradient, while in the region $x \in[0.24,0.61]$, the pressure gradient increases with an increase in $\mathrm{M}$ and decreases with an increase in, so a large pressure gradient is required to enable the flux to pass.

- The pressure gradient for different values of $\gamma$ and against $\mathrm{x}$ is displayed in Fig. 8. It is shown that for $x \in[0,0.23]$ and $x \in[0.6,1]$, the pressure gradient is large, while in the region $x \in[0.24,0.61]$, the pressure gradient decreases with an increase in $\gamma$.

\subsection{Velocity profile}

Figs. 9-12 are scrutinized to study the impacts of $M, \lambda 0, L$ and $\gamma$ on the axial velocity.

- We percept from Figs. 9-10 and 11 that the longitudinal velocity decreases with an increase in $M, \lambda 0$ and increases with an increase in $\gamma$ at the central region of the channel, whilst the longitudinal velocity increases with an increase in $M, \lambda 0$ and decreases with an increase in $\gamma$ at the Both sides of the channel regions.

- Fig 12. discuss the effect of slip parameter $\mathrm{L}$ on the longitudinal velocity, which divided into two parts, in the first half at the left channel the longitudinal velocity decreases with an increase in $\mathrm{L}$, the opposite effect of slip parameter can easily clarified at the second half at the right channel.

\subsection{Heat and mass characteristics}

Figs. 13-18 are depicted the influence of $\gamma, \mathrm{P}_{\mathrm{r}}, \mathrm{M}, \lambda_{1}$ and $L$ on the temperature $\theta(y)$.

- The influence of $\gamma, P_{r}$ and $\lambda 0$ are demonstrated in Figs. 13-14 and 17 higher values of $\gamma$ and $P_{r}$ yield increase in temperature $\theta(y)$, and $\theta(y)$ decreases with an increase in $\lambda 0$. Physically, larger heat generation parameter means an increase in heat produced inside the boundary layer which leads to higher temperature distribution. 
- Impact of slip parameter $L$ is portrayed in Fig. 18. It is disclosed that the temperature increases in the first part of the channel and then decreases in the second part of the channel with an increase in $L$.

- Figs. 13-14 investigated to display the difference effect of Hartmann number M on temperature $\theta$ (y) with absence of Joule heating term and in presence of Joule heating term. Firstly, here $\theta(y)$ reduces for higher values of Hartmann number $M$, with absence of Joule heating term. However, $\theta(y)$ has opposite effects with an increase Hartmann number $M$, in presence of Joule heating term. So it's important study the effect of Joule heating, its cause of leading to get more active due to stock of energy which is useful in enhancing temperature.

Figs. 19-25 are plotted to study the impact of $\gamma, P_{r}, M, \lambda_{1}, L$ and $S_{c}, S_{r}$ on the concentration $\varphi(\mathrm{y})$ :

- Figs. 19-24 are illustrated the influence of $\gamma, P_{r}, M, \lambda_{1}, L$ on concentration $\varphi(\mathrm{y})$, we will find an opposite effect on the effect of those parameter with the heat.

- Fig. 25 displayed to visualize that the concentration distribution decreases with an increases in $S_{c}, S_{r}$ together.

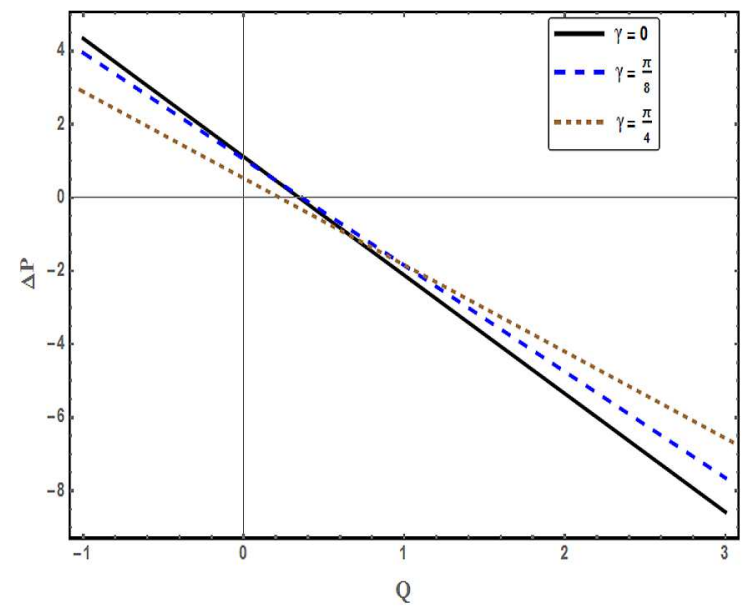

Fig. 1: The change of $\Delta \mathrm{P}$ with $\mathrm{Q}$ for several values of $\gamma$ at $\mathrm{a}=0.9, \mathrm{~b}=0.5, \mathrm{~d}=1.2, \phi=\frac{\pi}{3}, F_{r}=0.8, \mathrm{M}=0.5$, $\lambda 0=\frac{\pi}{4}, P_{r}=2, E_{c}=0.15, \mathrm{~L}=0, S_{r}=1, S_{c}=1, R_{e}=1$, $D_{f}=0, \alpha=0.2$.

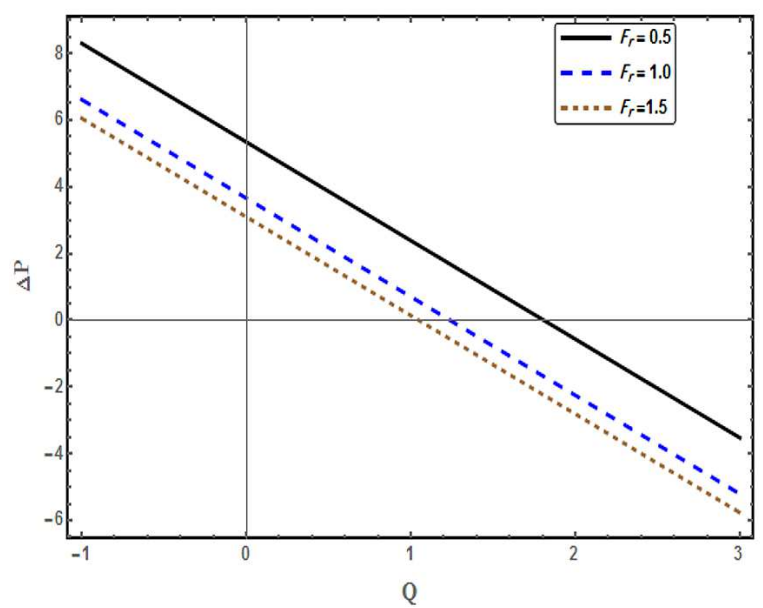

Fig. 3: The change of $\Delta \mathrm{P}$ with $\mathrm{Q}$ for several values of $F_{r}$ at $\mathrm{a}=0.9, \mathrm{~b}=0.5, \mathrm{~d}=1.2, \phi=\frac{\pi}{3}, \lambda 0=1, \mathrm{M}=1, \gamma=\frac{\pi}{4}, P_{r}=$ $3, E_{c}=0.15, \mathrm{~L}=0.1, S_{r}=1, S_{c}=1, R_{e}=1, D_{f}=0.2, \alpha=$ 1.

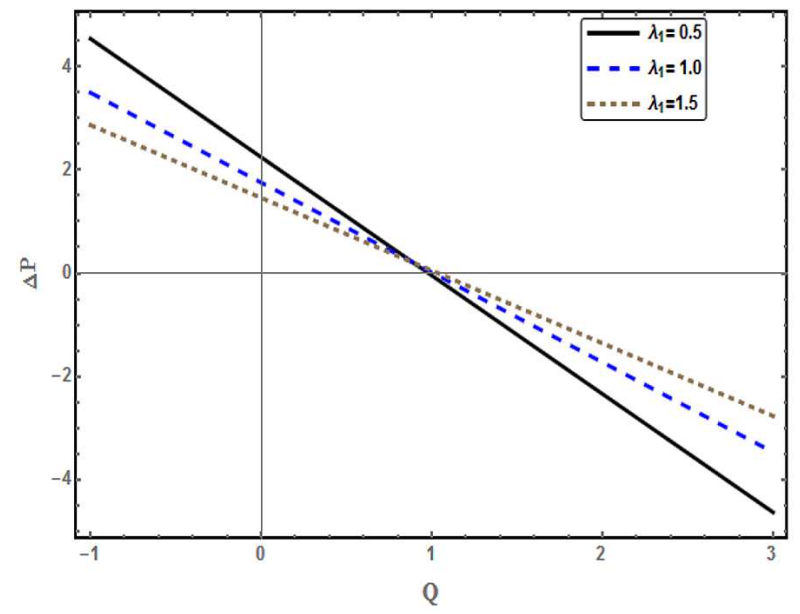

Fig. 2: The change of $\Delta \mathrm{P}$ with $\mathrm{Q}$ for several values of $\lambda 0$ at a=0.9, b=0.5, d=1.2, $\phi=\frac{\pi}{3}, F_{r}=0.8, \mathrm{M}=0.5$, $\gamma=\frac{\pi}{4}, P_{r}=2, E_{c}=0.15, \mathrm{~L}=0, S_{r}=1, S_{c}=1, R_{e}=1, D_{f}=$ $0, \alpha=0.2$.

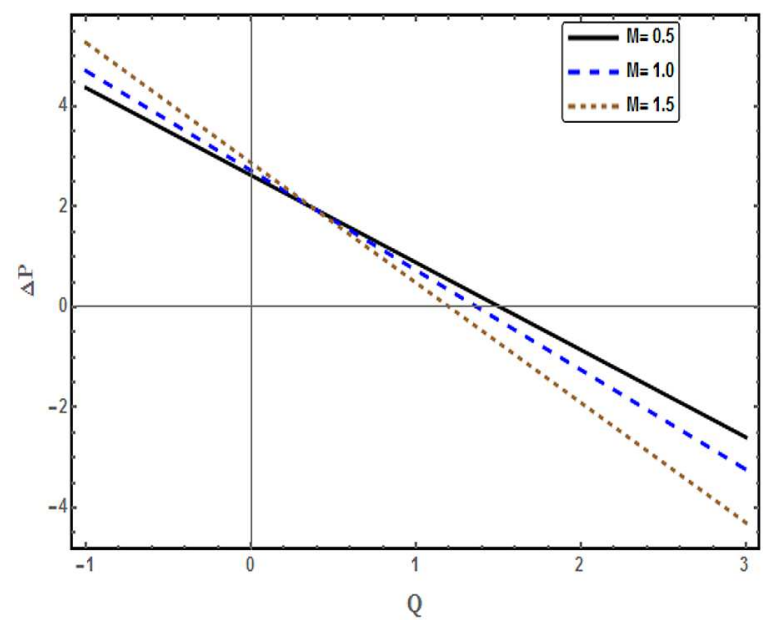

Fig. 4: The change of $\Delta \mathrm{P}$ with $\mathrm{Q}$ for several values of $M$ at a=0.9, b=0.5, d=1.2, $\phi=\frac{\pi}{3}, \lambda 0=1, F_{r}=1.5, \gamma=\frac{\pi}{4}$, $P_{r}=3, E_{c}=0.15, \mathrm{~L}=0.1, S_{r}=1, S_{c}=1, R_{e}=1, D_{f}=$ $0.2, \alpha=0.2$. 


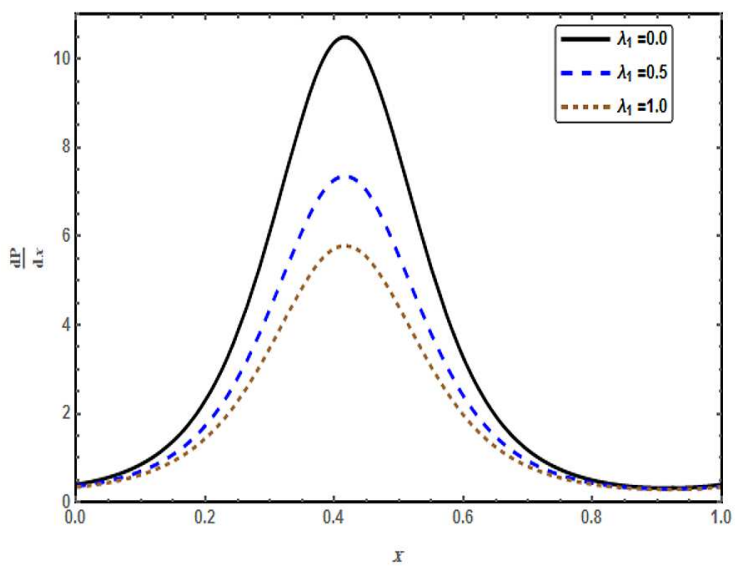

Fig. 5: The change of $\frac{d P}{d x}$ with $\mathrm{x}$ for several values of $\lambda 0$ at $\mathrm{a}=0.5, \mathrm{~b}=0.5, \mathrm{~d}=1.2, \phi=\frac{\pi}{3}, \mathrm{M}=1, \gamma=\frac{\pi}{4}, P_{r}=3, E_{c}=0.15$, $\mathrm{L}=0.1, S_{r}=1, S_{c}=1, R_{e}=1, D_{f}=0.2, \alpha=1, F_{r}=1, q=0.5$.

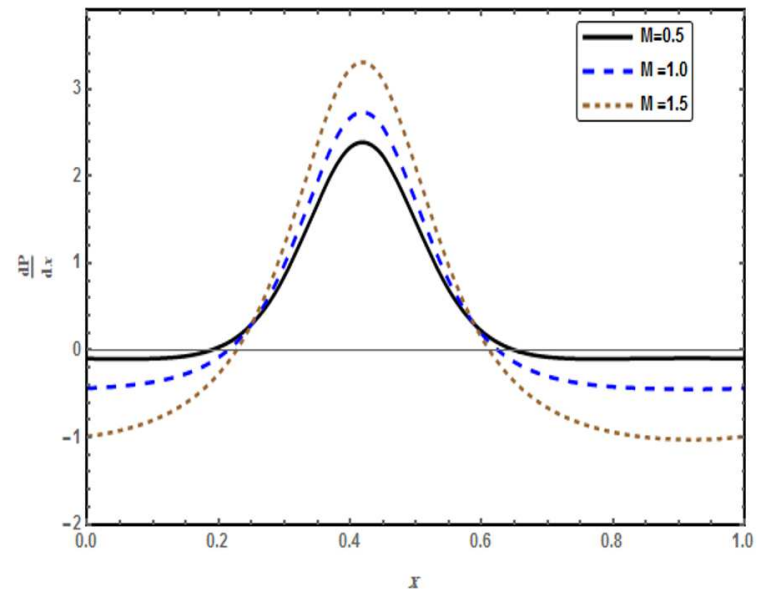

Fig. 7: The change of $\frac{d P}{d x}$ with $\mathrm{x}$ for several values of $M$ at $\mathrm{a}=0.9, \mathrm{~b}=0.5, \mathrm{~d}=1.2, \phi=\frac{\pi}{2}, F_{r}=0.8, \gamma=\frac{\pi}{8}, P_{r}=2, E_{c}=0.15$, $\mathrm{L}=0, S_{r}=1, S_{c}=1, R_{e}=0.5, D_{f}=0, \alpha=0.5, \lambda 0=1, q=0.5$

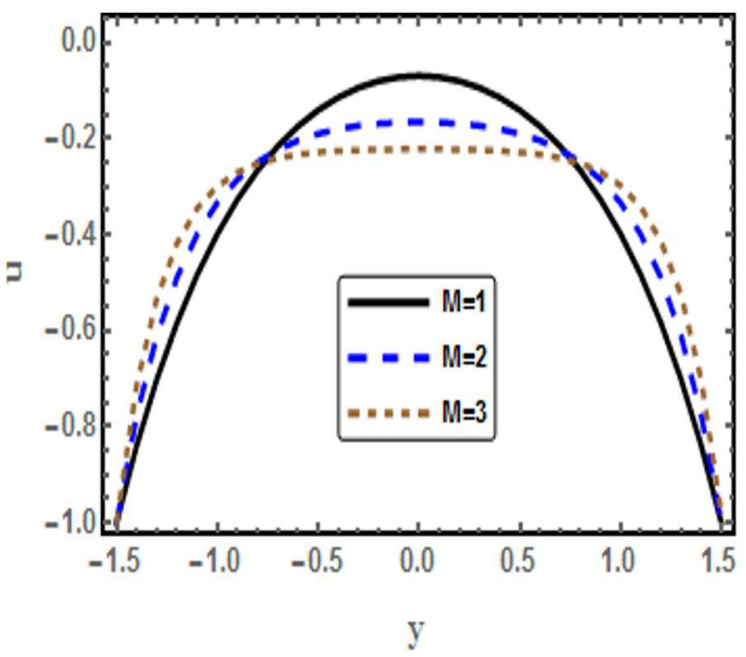

Fig. 9: The change of $u$ with $y$ for several values of $M$ at $\mathrm{a}=0.5, \mathrm{~b}=1.2, \mathrm{~d}=1.2, \phi=\frac{\pi}{2}, P_{r}=3, E_{c}=0.25, \mathrm{~L}=0.1, S_{r}=1$, $S_{c}=1, D_{f}=0.2, \lambda 0=1.5, q=-1, \gamma=\frac{\pi}{12}$.

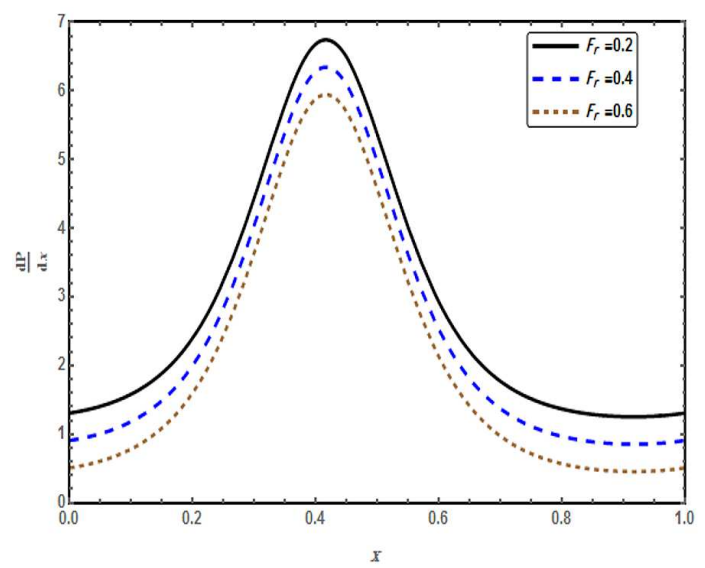

Fig. 6: The change of $\frac{d P}{d x}$ with $\mathrm{x}$ for several values of $F_{r}$ at $\mathrm{a}=0.5, \mathrm{~b}=0.5, \mathrm{~d}=1.2, \phi=\frac{\pi}{3}, \mathrm{M}=1, \gamma=\frac{\pi}{4}, P_{r}=3, E_{c}=0.15$, $\mathrm{L}=0.1, S_{r}=1, S_{c}=1, R_{e}=1, D_{f}=0.2, \alpha=1, \lambda 0=1, q=0.5$.

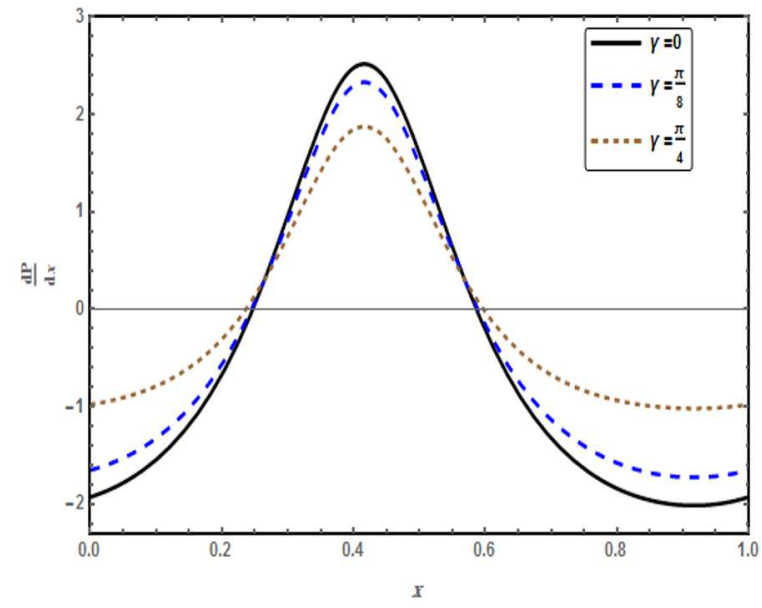

Fig. 8: The change of $\frac{d P}{d x}$ with $\mathrm{x}$ for several values of $\gamma$ at $\mathrm{a}=0.5, \mathrm{~b}=0.5, \mathrm{~d}=1.2, \phi=\frac{\pi}{2}, F_{r}=0.8, \mathrm{M}=1, P_{r}=2, E_{c}=0.15$, $\mathrm{L}=0, S_{r}=1, S_{c}=1, R_{e}=0.5, D_{f}=0, \alpha=0.5, \lambda 0=1, q=0.5$.

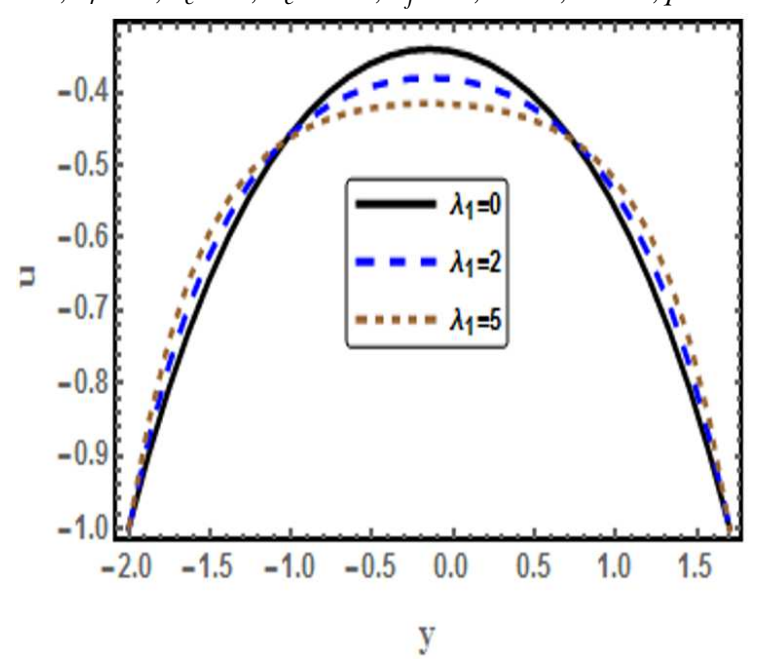

Fig. 10: The change of $u$ with $y$ for several values of $\lambda 0$ at $\mathrm{a}=0.7, \mathrm{~b}=1.2, \mathrm{~d}=1.2, \phi=\frac{\pi}{2}, \mathrm{M}=1, P_{r}=2, E_{c}=0.15, \mathrm{~L}=0.1$, $S_{r}=1, S_{c}=1, D_{f}=0.2, q=-2, \gamma=\frac{\pi}{12}$. 


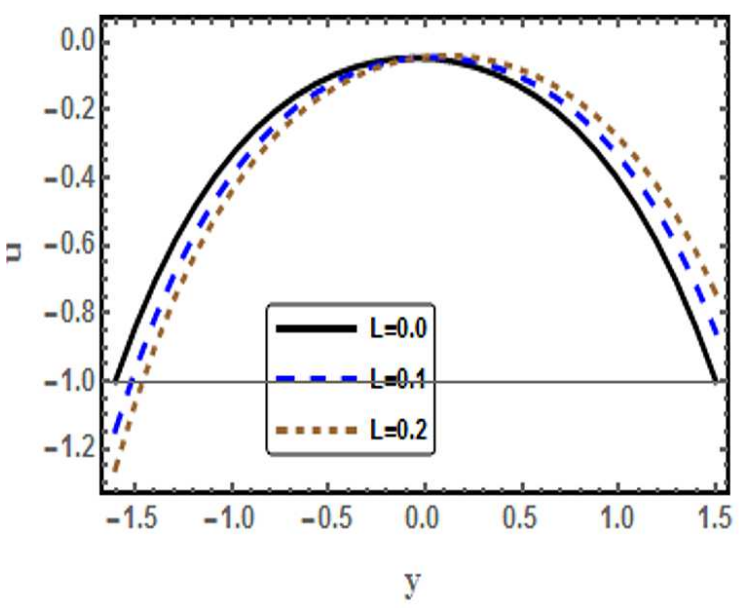

Fig. 11: The change of $u$ with $y$ for several values of $\mathrm{L}$ at a $=0.5, \mathrm{~b}=0.4, \mathrm{~d}=2, \phi=\frac{\pi}{3}, \mathrm{M}=1, P_{r}=2, E_{c}=0.15$, $\lambda 0=1, S_{r}=1, S_{c}=1, D_{f}=0.2, q=-1, \gamma=\frac{\pi}{12}$.

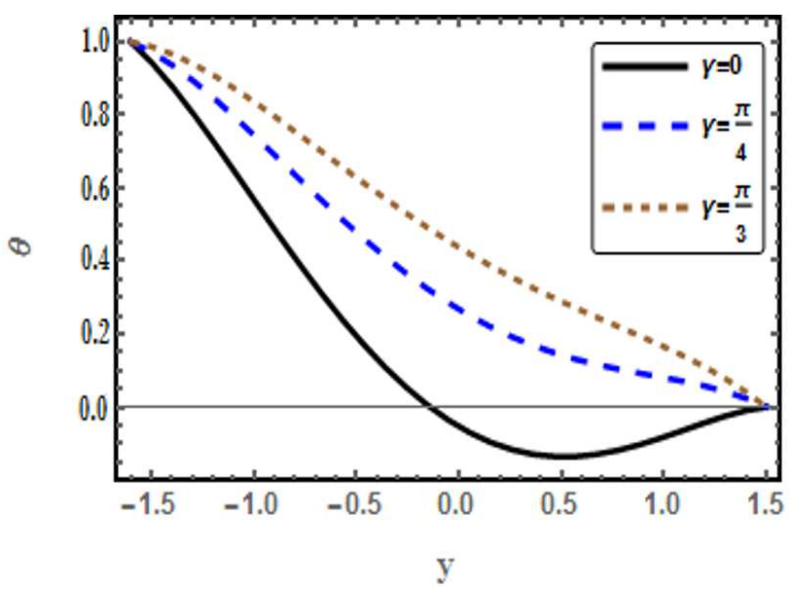

Fig. 13: The change of $\theta$ with $y$ for several values of $\gamma$ at $\mathrm{a}=0.5, \mathrm{~b}=0.4, \mathrm{~d}=2, \phi=\frac{\pi}{3}, \mathrm{M}=1, P_{r}=3, E_{c}=0.15$, $\lambda 0=1, S_{r}=1, S_{c}=1, D_{f}=0.2, L=0.1, q=-1$.

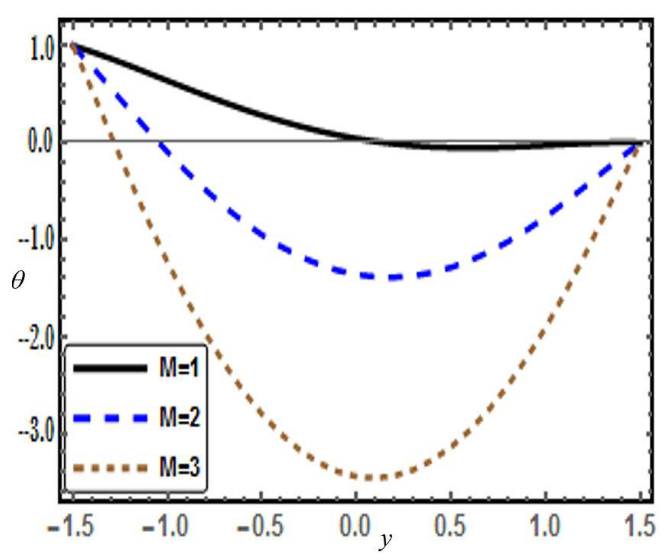

Fig. 15: The change of $\theta$ with $y$ for several values of $M$ at a $=0.5, \mathrm{~b}=1.2, \mathrm{~d}=1.2, \phi=\frac{\pi}{2}, P_{r}=3, E_{c}=0.25, \mathrm{~L}=0$, $S_{r}=1, S_{c}=1, D_{f}=0, \lambda 0=1.5, q=-1, \gamma=\frac{\pi}{12}$ (With absence of Joule heating term).

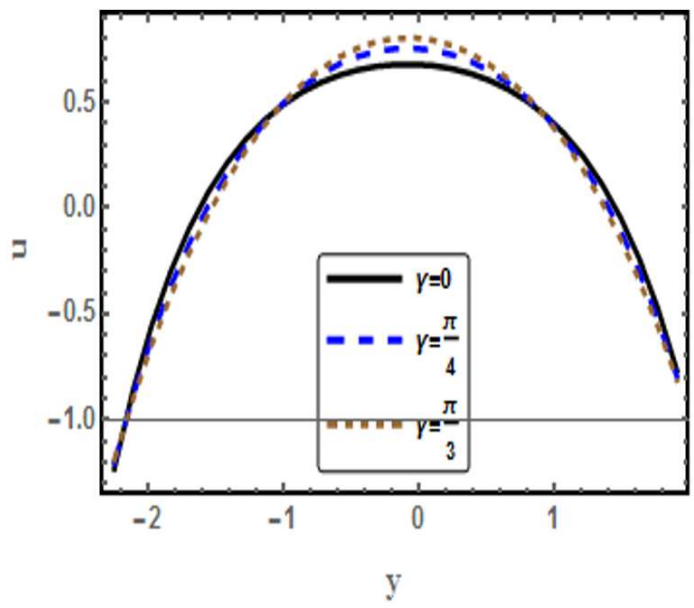

Fig. 12: The change of $u$ with $y$ for several values of $\gamma$ at $\mathrm{a}=0.5, \mathrm{~b}=0.4, \mathrm{~d}=2, \phi=\frac{\pi}{3}, \mathrm{M}=1, P_{r}=3, E_{c}=0.15$, $\lambda 0=1, S_{r}=1, S_{c}=1, D_{f}=0.2, L=0.1, q=-1$.

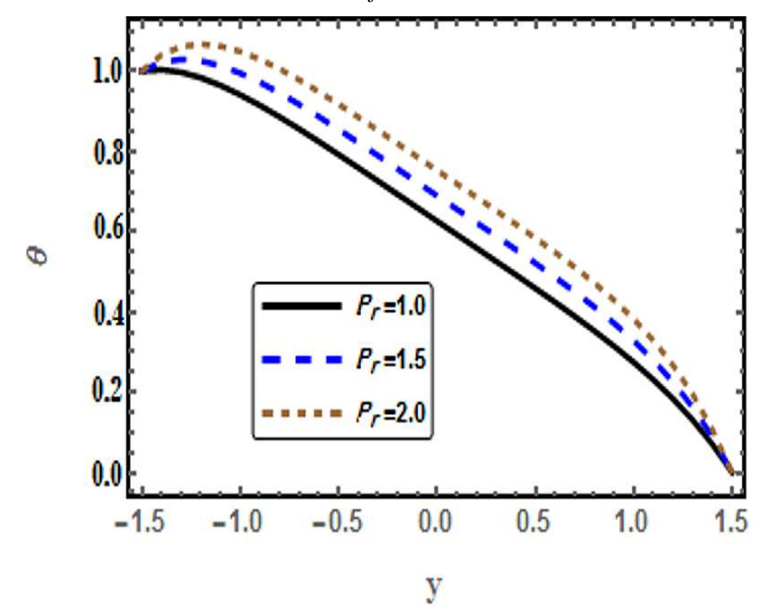

Fig. 14: The change of $\theta$ with $y$ for several values of $P_{r}$ at $\mathrm{a}=0.5, \mathrm{~b}=1.2, \mathrm{~d}=1.5, \phi=\frac{\pi}{2}, \mathrm{M}=1, \gamma=\frac{\pi}{8}, E_{c}=$ $0.15, \lambda 0=1, S_{r}=1, S_{c}=1, D_{f}=0, L=0, q=-1$.

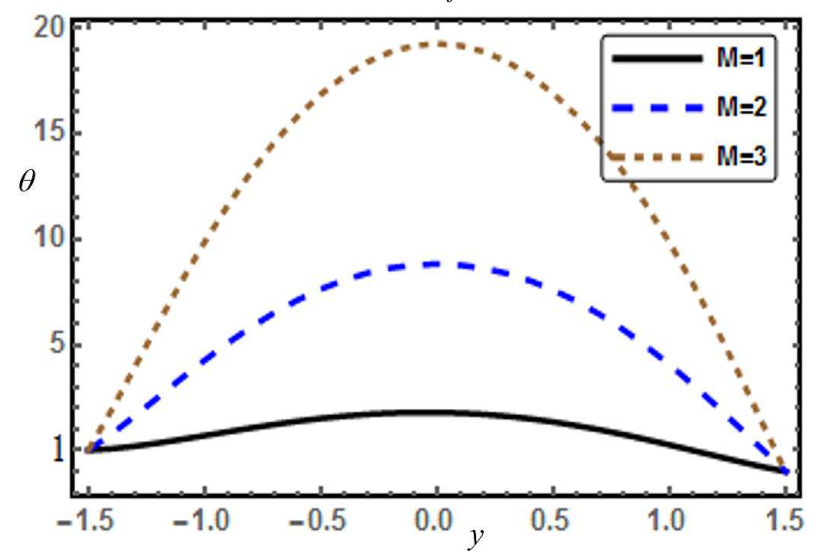

Fig. 16: The change of $\theta$ with $y$ for several values of $M$ at $\mathrm{a}=0.5, \mathrm{~b}=1.2, \mathrm{~d}=1.2, \phi=\frac{\pi}{2}, P_{r}=3, E_{c}=0.25, \mathrm{~L}=0.1$, $S_{r}=1, S_{c}=1, D_{f}=0.2, \lambda 0=1.5, q=-1, \gamma=\frac{\pi}{12}$ (In presence of Joule heating term). 


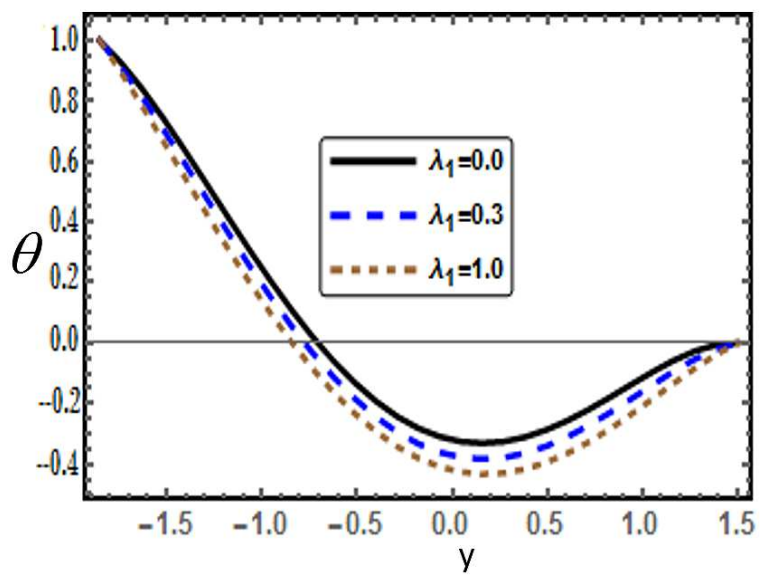

Fig. 17: The change of $\theta$ with $y$ for several values of $\lambda 0$ at $\mathrm{a}=0.7, \mathrm{~b}=1.2, \mathrm{~d}=1.2, \phi=\frac{\pi}{2}, \mathrm{M}=1, P_{r}=2, E_{c}=$ $0.15, \mathrm{~L}=0.1, S_{r}=1, S_{c}=1, D_{f}=0.2, q=-2, \gamma=\frac{\pi}{12}$.

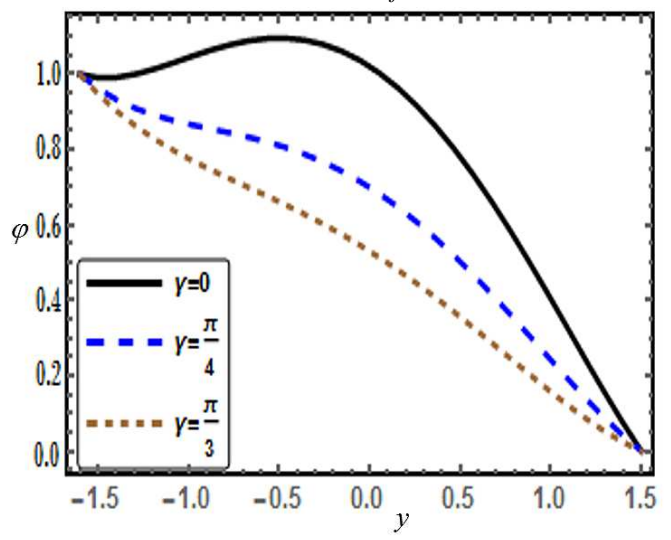

Fig. 19: Fig 19. The change of $\varphi$ with $y$ for several values of $\gamma$ at $\mathrm{a}=0.5, \mathrm{~b}=0.4, \mathrm{~d}=2, \phi=\frac{\pi}{3}, \mathrm{M}=1, P_{r}=3$, $E_{c}=0.15, \lambda 0=1, S_{r}=1, S_{c}=1, D_{f}=0.2, L=0.1$, $q=-1$.

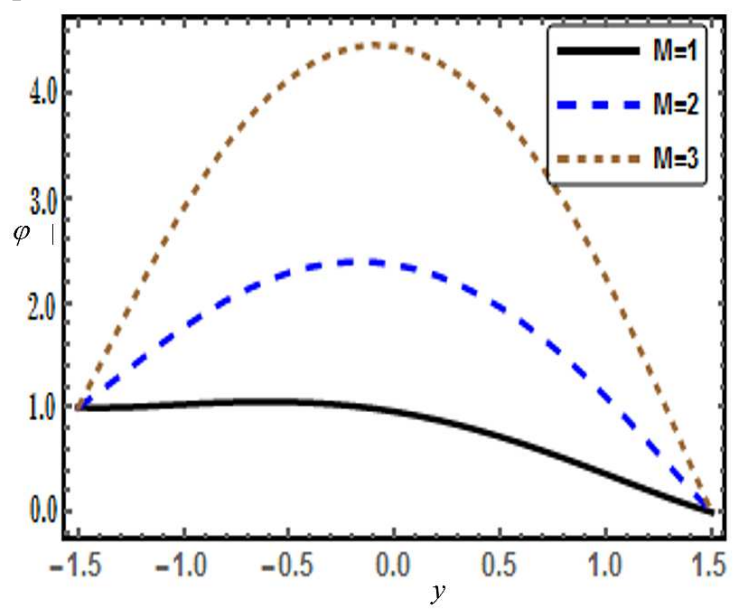

Fig. 21: Fig 19. The change of $\varphi$ with $y$ for several values of $M$ at a= $0.5, \mathrm{~b}=1.2, \mathrm{~d}=1.2, \phi=\frac{\pi}{2}, P_{r}=3, E_{c}=$ $0.25, \mathrm{~L}=0, S_{r}=1, S_{c}=1, D_{f}=0, \lambda 0=1.5, q=-1$, $\gamma=\frac{\pi}{12}$ (With absence of Joule heating term).

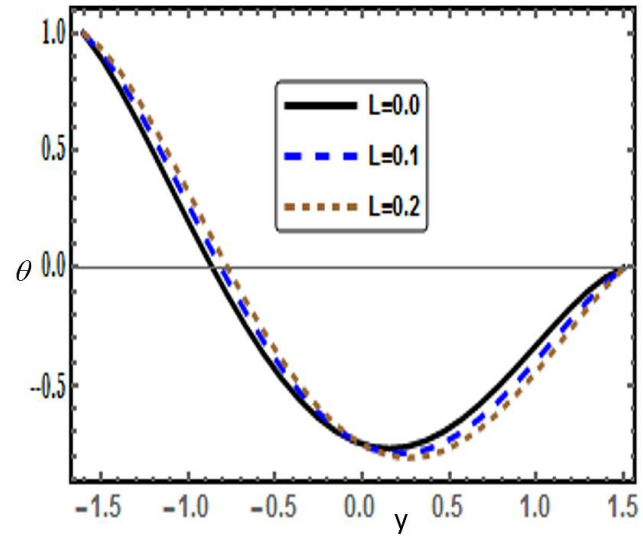

Fig. 18: The change of $\theta$ with $y$ for several values of $\mathrm{L}$ at $\mathrm{a}=0.5, \mathrm{~b}=0.4, \mathrm{~d}=2, \phi=\frac{\pi}{3}, \mathrm{M}=1, P_{r}=2, E_{c}=0.15$, $\lambda 0=1, S_{r}=1, S_{c}=1, D_{f}=0.2, q=-1, \gamma=\frac{\pi}{12}$.

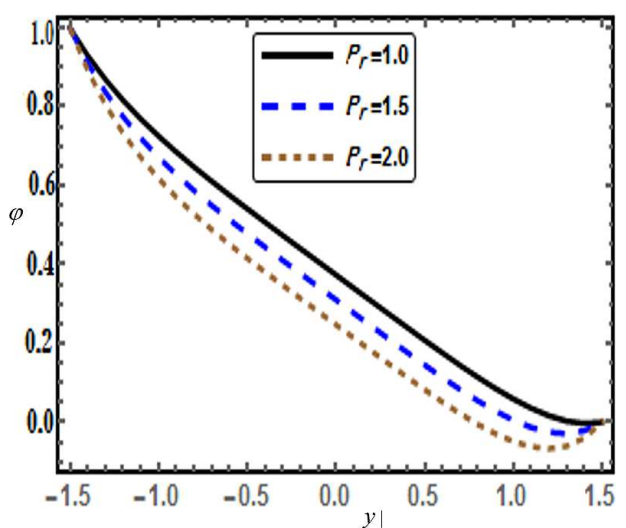

Fig. 20: The change of $\varphi$ with $y$ for several values of $P_{r}$ at $\mathrm{a}=0.5, \mathrm{~b}=1.2, \mathrm{~d}=1.5, \phi=\frac{\pi}{2}, \mathrm{M}=1, \gamma=\frac{\pi}{8}, E_{c}=$ $0.15, \lambda 0=1, S_{r}=1, S_{c}=1, D_{f}=0, L=0, q=-1$.

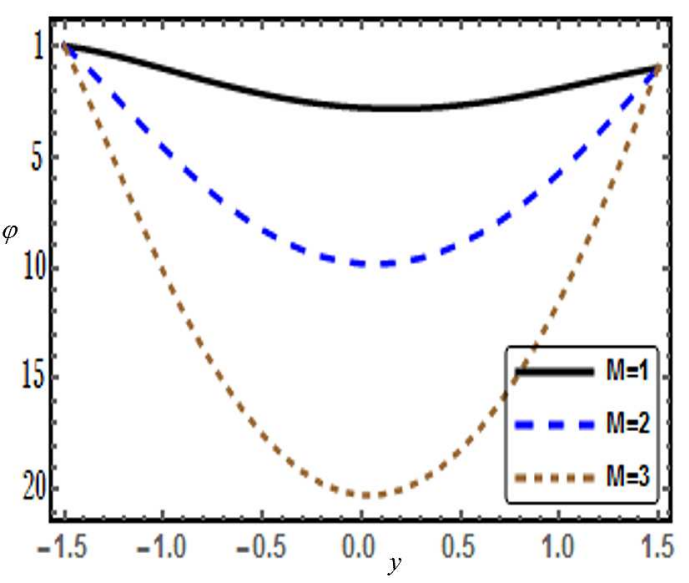

Fig. 22: The change of $\varphi$ with $y$ for several values of $M$ at a $=0.5, \mathrm{~b}=1.2, \mathrm{~d}=1.2, \phi=\frac{\pi}{2}, P_{r}=3, E_{c}=0.25, \mathrm{~L}=0.1$, $S_{r}=1, S_{c}=1, D_{f}=0.2, \lambda 0=1.5, q=-1, \gamma=\frac{\pi}{12}($ In presence of Joule heating term). 


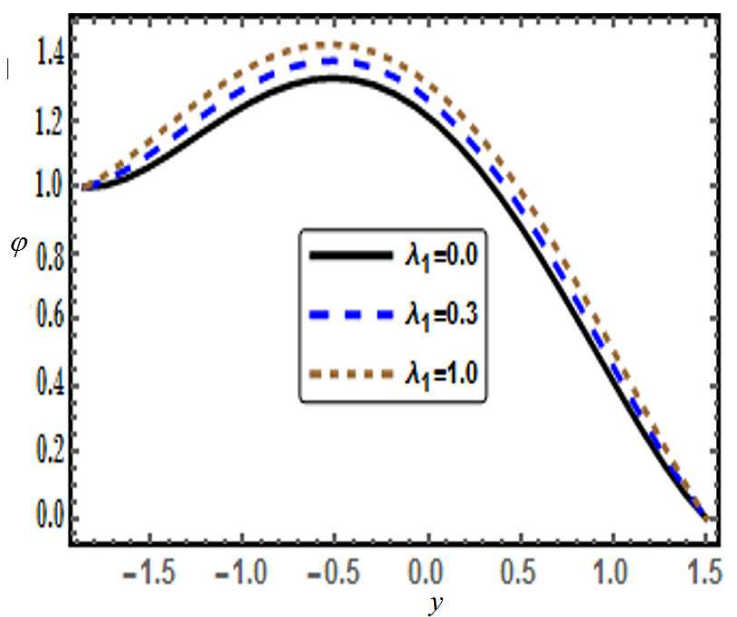

Fig. 23: Fig 19. The change of $\varphi$ with $y$ for several values of $\lambda 0$ at $\mathrm{a}=0.7, \mathrm{~b}=1.2, \mathrm{~d}=1.2, \phi=\frac{\pi}{2}, \mathrm{M}=1$, $P_{r}=2, E_{c}=0.15, \mathrm{~L}=0.1, S_{r}=1, S_{c}=1,{ } D_{f}=0.2$, $q=-2, \gamma=\frac{\pi}{12}$.

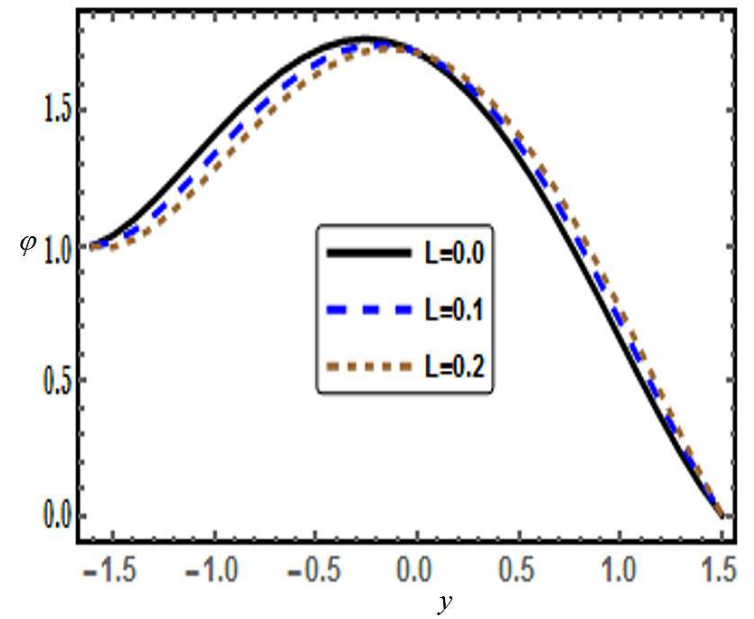

Fig. 24: The change of $\varphi$ with $y$ for several values of $L$ at $\mathrm{a}=0.5, \mathrm{~b}=0.4, \mathrm{~d}=2, \phi=\frac{\pi}{3}, \mathrm{M}=1, P_{r}=2, E_{c}=0.15$, $\lambda 0=1, S_{r}=1, S_{c}=1, D_{f}=0.2, q=-1, \gamma=\frac{\pi}{12}$.

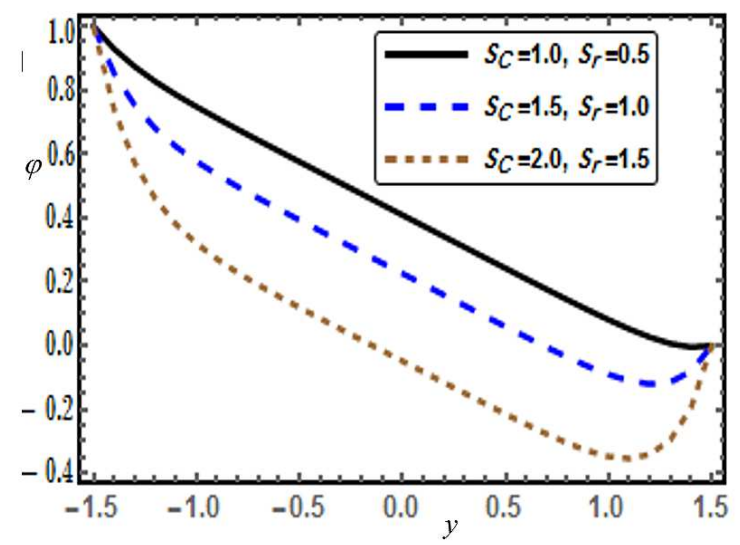

Fig. 25: The change of $\varphi$ with $y$ for several values of $S_{r}$ and $S_{c}$ at $a=0.5, b=1.2, d=2, \phi=\frac{\pi}{2}, \mathrm{M}=1, P_{r}=2$, $\mathrm{L}=0, E_{c}=0.15, \lambda 0=1, D_{f}=0.2, q=2, \gamma=\frac{\pi}{8}$.

Table 2: Comparison of velocity, Temperature and Concentration profiles for the selected values of the embedded parameters $M=1, P_{r}=3, \gamma=\frac{\pi}{3}, E_{c}=0.25, L=0.1, \phi=\frac{\pi}{4}, d=1.2, D_{f}=0.2, S_{r}=1, S_{c}=1, a=0.4, b=0.5$.

\begin{tabular}{|c|c|c|c|c|c|c|c|c|c|}
\hline$y$ & $\begin{array}{l}u(y) N D \\
\text { Solve }\end{array}$ & $u(y) M s-D T M$ & $\begin{array}{l}\begin{array}{l}\text { Error } \\
\text { solutions }\end{array} \\
\text { of }\end{array}$ & $\begin{array}{l}\theta(y) N D \\
\text { Solve }\end{array}$ & $\begin{array}{l}\theta(y) \quad M s- \\
D T M\end{array}$ & $\begin{array}{ll}\text { Error } & \text { of } \\
\text { solutions }\end{array}$ & $\begin{array}{l}\varphi(y) N D \\
\text { Solve }\end{array}$ & $\varphi(y) M s-D T M$ & $\begin{array}{ll}\text { Error } \\
\text { solutions }\end{array}$ \\
\hline-1.55355 & -1.02585761 & -1.0258572 & 0 & 0 & 0 & 0 & 0 & 0 & 0 \\
\hline-1.25819 & -1.09809064 & -1.09809062 & $2 \times 10^{-8}$ & 0.231895 & 0.231895 & $2 \times 10^{-9}$ & 0.00165098 & 0.00165099 & $1 \times 10^{-9}$ \\
\hline-0.9628 & -1.02684131 & -1.02684 & $2 \times 10^{-8}$ & 0.451063 & 0.451063 & $1 \times 10^{-8}$ & 0.00980551 & 0.0098055 & $6 \times 10^{-9}$ \\
\hline-0.66748 & -0.84418128 & -0.844181 & $4 \times 10^{-8}$ & 0.650584 & 0.650584 & $2 \times 10^{-8}$ & 0.0284813 & 0.0284813 & $1 \times 10^{-8}$ \\
\hline-0.37213 & -0.59130465 & -0.591305 & $2 \times 10^{-8}$ & 0.817079 & 0.817079 & $4 \times 10^{-8}$ & 0.0656345 & 0.0656345 & $2 \times 10^{-8}$ \\
\hline-0.07677 & -0.31896831 & -0.318968 & $6 \times 10^{-8}$ & 0.9428577 & 0.942858 & $7 \times 10^{-8}$ & 0.127577 & 0.127577 & $4 \times 10^{-8}$ \\
\hline 0.218578 & -0.08524376 & -0.0852439 & $1 \times 10^{-7}$ & 1.03005 & 1.03005 & $1 \times 10^{-7}$ & 0.217309 & 0.217309 & $6 \times 10^{-8}$ \\
\hline 0.513933 & 0.04705016 & 0.04705 & $1 \times 10^{-1}$ & 1.08787 & 1.08787 & $1 \times 10^{-1}$ & 0.336106 & 0.336106 & $9 \times 10^{-8}$ \\
\hline 0.809289 & 0.00960650 & 0.00960633 & $1 \times 10^{-7}$ & 1.12281 & 1.12281 & $2 \times 10^{-7}$ & 0.488515 & 0.488515 & $1 \times 10^{-7}$ \\
\hline 1.104644 & -0.27344174 & -0.273442 & $2 \times 10^{-8}$ & 1.11879 & 1.11879 & $2 \times 10^{-1}$ & 0.692632 & 0.692632 & $1 \times 10^{-7}$ \\
\hline 1.399999 & -0.87068411 & -0.870685 & $5 \times 10^{-8}$ & 1. & 1. & $3 \times 10^{-7}$ & 1 & 1 & $2 \times 10^{-7}$ \\
\hline
\end{tabular}




\section{Conclusions}

Joule heating effects on peristaltic flow of Jeffrey fluid in presence of heat and mass transfer in an inclined symmetric or asymmetric channel is analyzed. Main findings of present analysis are listed below.

- Pressure gradient tends to decrease with an increase in the Jeffery parameter and Hartmann number. However, reverse behavior is noted for the angle of inclination.

- High of Jeffrey parameter and Hartmann number leads to increase the longitudinal velocity.

- There is an enhancement of temperature for large angle of inclination and Prandtl number.

- The concentration field increases with an increase in $M$ and decreases with an increase in $S_{c}$ and $S_{r}$ as in [1].

- In the center of the channel, the pressure gradient increases with an increase in $M$ and decreases with the increase in $\gamma$ as in [1].

- Effect of Hartmann number in temperature and concentration in presence of joule heating term is opposite effect in absence of joule heating.

- A fine comparison of our results and obtainable results available in the limiting case are also offered in the table.

- In fact, the multi-step DTM is applicable to nonlinear models such as Non-Newtonian peristaltic fluid models which is more complicated and have a higher degree of non-linearity, in a direct way without using linearization, perturbation or restrictive assumptions.

\section{Competing interests}

The authors declare that they have no competing interests.

\section{Authors' contributions}

All authors have contributed to all parts of the article. All authors read and approved the final manuscript.

\section{References}

[1] S. Nadeem and S. Akram, Influence of inclined magnetic field on peristaltic flow of a Jeffrey fluid with heat and mass transfer in a n inclined symmetric or asymmetric channel, Asia-Pacific Journal of Chemical Engineering, 7 (2012) 33-44.

[2] T. Hayat, Q. Hussain, M. U. Qureshi, N. Ali and A. A. Hendi, Influence of slip condition on the peristaltic transport in an asymmetric channel with heat transfer: An exact solution, International Journal for Numerical Methods in Fluids, 67 (2011) 1944-1959.

[3] S.Srinivas and R.Muthuraj, Effects of chemical reaction and space porosity on MHD mixed convective flow in a vertical asymmetric channel with peristalsis, Mathematical and Computer Modeling, 54 (2011) 1213-1227.

[4] N. T. Eldabe, M.A. Elogail, S.M. Elshaboury and A. A. Hasan, Hall effects on the peristaltic transport of Williamson fluid through a porous medium with heat and mass transfer, Applied Mathematics and Modeling, 40 (2016) 315-328.

[5] T. Hayat, Z. Asghar, S. Asghar and S. Mesloub, Influence of inclined magnetic field on peristaltic transport of fourth grade fluid in an inclined asymmetric channel, Journal of the Taiwan Institute of Chemical Engineers, 41 (2010) 553-563

[6] T. Hayat , M. Iqbal ,H. Yasmin and F, Alsaadi, Hall effects on peristaltic flow of couple stress fluid in an inclined asymmetric channel, International Journal of Biomathematics, 7 (2014) 1450057- 1: 1450057- 34.

[7] V.P. Rathod and M. Mahadev, Peristaltic Flow of Jeffrey Fluid with Slip Effects in an Inclined Channel, Journal of Chemical, Biological and Physical Sciences, 2 (2012) 1987-1997.

[8] T. Hayat, N. Ahmad and N. Ali, Effects of an endoscope and magnetic field on the peristalsis involving Jeffrey fluid, Communications in Nonlinear Science and Numerical Simulation 13, (2008), 1581-1591. 
[9] N. Sher Akbar, S. Nadeem and C. Lee, Characteristics of Jeffrey fluid model for peristaltic flow of chyme in small intestine with magnetic field, Results in Physics, 3 (2013) 152-160.

[10] T. Hayat, M. Waqas, M. I. Khan and A. Alsaedi, Impacts of constructive and destructive chemical reactions in magneto hydrodynamic (MHD) flow of Jeffrey liquid due to nonlinear radially stretched surface, Journal of Molecular Liquids, 225 (2017) 302-310.

[11] Q. Hussain, S. Asghar, T. Hayat and A. Al Saedi, Heat transfer analysis in peristaltic flow of MHD Jeffrey fluid with variable thermal conductivity, Advances in Applied Mathematics and Mechanics, 36 (2015) 499-516.

[12] T. Hayat, F. M. Abbasi, M. Al Yami and S. Monaquel, Slip and Joule heating effects in mixed convection peristaltic transport of nanofluid with Soret and Dufour effects, Journal of Molecular Liquids, 194 (2014) 93-99.

[13] T. Hayat, M. Rafiq, A. Alsaedi and B. Ahmad, Radiative and Joule heating effects on peristaltic transport of dusty fluid in a channel with wall properties, The European Physical Journal Plus, 9 (2014) 129-225.

[14] M. G. Reddy and K. V. Reddy, Influence of Joule Heating on MHD Peristaltic Flow of a Nanofluid with Compliant Walls, Procedia Engineering, 127, (2015), $1002-1009$.

[15] S. Abzal, S. Varma and S. Ravi Kumar, Influence of slip and Joule heating with radiation on MHD peristaltic blood flow with porous medium through a coaxial asymmetric vertical tapered channel blood flow analysis study, International Journal of Advanced Science and Technology, 91 (2016) 71-90.

[16] T. Hayat, A. Alsaedi, M. Rafiq and B. Ahmad, Joule heating and thermal radiation effects on peristalsis in curved configuration, Results in Physics, 6 (2016) 1088-1095.

[17] R. A. Zait, A.A. El-Shekhipy and N. M. Abdo, Statistical measures approximations for the Gaussian part of the stochastic nonlinear damped Ruffing oscillator solution process under the application of Wiener Hermite expansion linked by the multi-step differential transformed method, Journal of the Egyptian Mathematical Society, (2016) 1-12.

[18] M. Hatami and D. D. Ganji, Motion of a spherical particle on a rotating parabola using Lagrangian and high accuracy Multi-step Differential Transformation Method, Powder Technology, 258 (2014) 94-98.

[19] M. Nourifar, A. A. Sani and A. Keyhani, Efficient multi-step differential transform method: Theory and its application to nonlinear oscillators, Communications in Nonlinear Science and Numerical Simulation, 53 (2017) 154-183.

[20] A. Yildirim, A. Gkdogan, and M. Merdan, Chaotic systems via multi-step differential transformation method, Canadian Journal of Physics, 90 (2012) 391 - 406.

[21] H. Ockendon and J. R. Ockendon, Variable-viscosity flows in heated and cooled channels, Journal of Fluid Mechanics, 83 (1977) 177-190.

[22] N. T. M. Eldabe, B. M. Agoor and H. Alame, Peristaltic motion of Non-Newtonian fluid with heat and mass transfer through a porous medium in channel under uniform magnetic field, journal of fluids, ID 525769 (2014) 1-12. 